\title{
Challenges faced in choosing novel targets to treat chronic pain
}

"...the growing body of knowledge indicates that chronic pain is a complex experience arising from the intricate interaction of external stimuli, the neurochemical and structural properties of the peripheral and central nervous systems, the organism's prior experiences and the current emotional state."

Chronic pain is a widely experienced problem in the USA, with estimates of its prevalence reaching as high as $50 \%$. Importantly, in one study, approximately a third of sufferers reported that their pain was 'disabling' and interfered with their ability to work and enjoy a normal life [1]. Thus, despite the presence of a number of currently available pain therapeutics [2], there is a clear need for additional effective and safe treatments for chronic pain.

“...despite the presence of a number of currently available pain therapeutics, there is a clear need for additional effective and safe treatments for chronic pain."

The first step in the rational development of a novel, safe and effective treatment is to identify a specific molecular substrate, which when selectively targeted will provide relief from chronic pain without intolerable side effects. Once a target is selected, the pharmaceutical industry is generally very effective in developing a molecule that achieves the desired function at the molecular level. However, achieving this milestone does not guarantee success. Therefore, while conceptually it would be best if we could develop and clinically test compounds that target every one of the possible biological substrates, the cost of developing new drugs requires us to select a limited subset for active work. One of the most important and challenging aspects of analgesic drug development is to choose which of the many possible biological mechanisms proposed to be involved in pain [3-5] should be pursued.

An appreciation of this challenge begins with an understanding of the basic properties of the pain processing system in animals. First, it is important to remember that pain plays an important role in protecting an animal from damage that can result from a variety of insults, blunt traumas, puncture wounds, temperature extremes and exposure to dangerous toxic substances. It is not surprising that animals have evolved sophisticated ways to detect, process and remember painful stimuli that are associated with these environmental dangers.

The biology of a normal protective pain experience can be broken down into three mechanistic categories that are distributed throughout the nervous system (Figure I). The first category comprises mechanisms involved in the detection or transduction of painful stimuli by the peripheral nervous system. The second category is best described as transmission mechanisms that propagate the pain signal through the pain circuitry. The third and final mechanistic category, which is mainly localized to the CNS, is the neuronal processing that modulates the pain signal as it propagates through the pain circuitry and leads to the ultimate integrated conscious experience of pain.

In light of these three categories, we can then consider the challenges, pros and cons of pursuing individual targets. Specifically, what are the theoretical benefits and risks inherent in preventing the initiation of the pain signal by blocking the transduction event versus modulating the transmission and propagation of the pain signal through the pain circuitry versus altering the processing of the pain signals in the CNS?

\section{Targeting pain signal transduction}

Recent years have witnessed an explosion in the understanding of the mechanisms that transduce painful stimuli [3-6]. A large family of channels, collectively called the transient receptor potential (TRP) channels, appear to play a major role in sensory and pain transduction [5]. The TRP V1

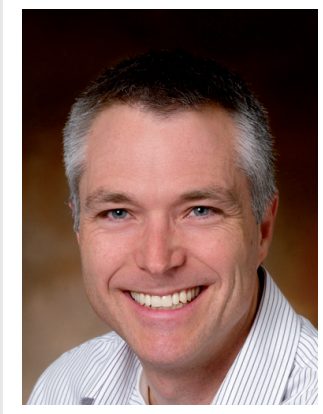

\section{Darrell A Henze}

Department of Pain Research, Merck Research Laboratories, 770 Sumneytown Pike, PO Box 4, WP26A-2000, West Point, PA 19486-0004, USA

Tel.: +I 2156529093

Fax: +| 215993 |40|

E-mail: darrell_henze@ merck.com

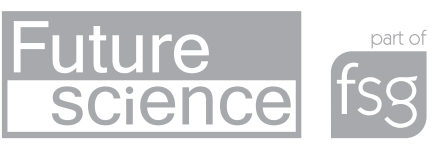




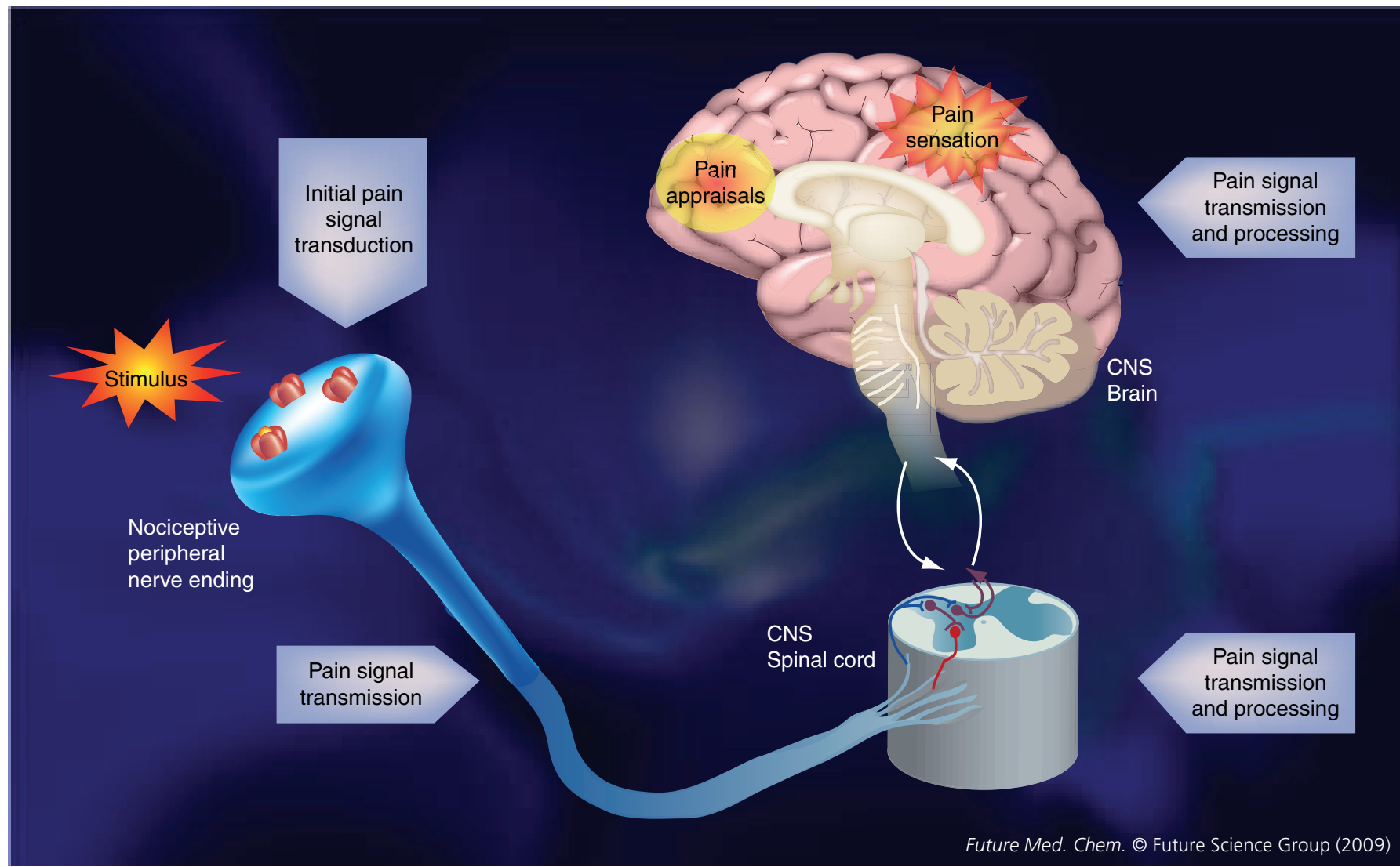

Figure 1. Pain circuitry, showing the localization of the three mechanistic categories of potential analgesic targets.

Typically, pain is first transduced or detected at the nociceptive peripheral nerve endings. The pain signal then propagates into the spinal cord and upward towards the brain via a collection of transmission mechanisms that underlie action potential propagation and synaptic transmission. Once the pain signal is in the CNS, a third series of mechanisms modulate and process the pain signal to generate the final integrated conscious experience of pain.

channel is expressed in nociceptive nerve endings and is known to be activated by the hot temperatures associated with pain. The TRPV1 channel also responds to acid as well as capsaicin, which is the pungent ingredient in hot peppers [7]. Other TRP channels, TRPV2, -3 and -4 , reportedly can also help detect hot or warm temperatures. Another member of the TRP family, TRPA1, is emerging as a central player in the detection of pain associated with many noxious chemicals and may also help detect painful mechanical stimuli [8]. A different family of channels, the acid-sensing ion channels, detect acid and thus show redundancy with the acid sensing properties of the TRPV1 channel [9]. There are also a variety of other receptors on nociceptive nerve endings with the ability to detect substances that are all generally elevated by inflammation, including bradykinin, the neuropeptide substance $\mathrm{P}$, prostaglandins, ATP, calcitonin gene related peptide(CGRP) and various chemokines [3]. As might be expected, the majority of these receptors and inflammatory mediators are currently being pursued by the pharmaceutical industry for pharmaceutical intervention with the simple goal of blocking the initial transduction event underlying chronic pain. To date, there is no marketed analgesic that is known to selectively target a pain transduction mechanism.

There are two major challenges faced when selectively targeting individual molecular transducers of pain. The first is that a significant functional redundancy exists in these molecular targets. Therefore, a key challenge is whether any one of these molecular targets plays a significant enough role across the spectrum of chronic pain states such that selective pharmacological intervention will provide clinically meaningful pain relief. In addition, there are some forms of chronic pain, so-called central pain, that do not involve a peripheral transduction event, but instead result from aberrant spontaneous activity within the CNS. The second challenge concerns whether, in the process of blocking chronic pain, 
a pharmacological intervention will also blunt a person's ability to respond in a self-protective manner to an acute noxious stimulus. A significant loss of protective pain responses has the potential to be an important safety limitation for a chronic pain treatment.

\section{Targeting pain signal transmission}

The second mechanistic category, transmission, plays a role after pain signal transduction occurs and action potentials are initiated in the nociceptive nerve endings. The action potentials then propagate along the nerves, through the dorsal root ganglia, into the spinal cord and, finally, evoke neurotransmitter release onto projection neurons and interneurons of the dorsal horn. The process of action-potential initiation, propagation and synaptic transmission is repeated for every neuron involved in the CNS pain circuitry. There are a number of potential molecular targets that could potentially interfere with the propagation of the pain signal into the spinal cord and throughout the CNS. These include a variety of voltagegated sodium, potassium and calcium ion channels that underlie the action potential and trigger the release of neurotransmitters from presynaptic terminals. The currently available analgesic treatments topical lidocaine and intrathecal ziconitide are examples of targeting sodium and calcium channels, respectively. A variety of ligand-gated receptor ion channels, such as glutamate receptors, are also critical to the synaptic transmission that propagates the pain signal through the CNS.

A potential advantage of targeting a transmission mechanism is that it might be possible to impact the propagation of all incoming pain signals regardless of the specifics of the initial stimuli, increasing the chance that a single compound will have efficacy against all types of pain. However, the basic molecular mechanisms underlying the transmission of the pain signal are shared as common mechanistic building blocks that underlie almost all neuronal function. Thus, the greatest challenge in targeting molecular mechanisms involved in pain signal transmission is achieving analgesic efficacy without generating intolerable side effects due to a broad disruption of CNS function or a significant blunting of nonpainful sensation. The success of targeting a transmission mechanism will depend on identifying specific subtypes of channels or receptors that are enriched in expression in the pain circuitry. For example, some of the most hotly pursued targets for a novel pain therapy are the voltage-gated sodium channels, specifically subtypes 1.3, 1.7, 1.8 and 1.9 [10].
The selective expression or pain-induced upregulation of these sodium channel subtypes in the axons of nociceptors provides hope that selective inhibition of one or more of these subtypes might provide a safe and effective analgesic therapy, without a widespread impact on neuronal function.

\section{Targeting pain signal processing}

The final mechanistic category of pain targets are those that are involved in the complex neuronal processing that modulates and integrates the pain signal in the CNS circuitry. One currently available treatment that likely acts, at least in part, by modulating pain signal processing in the CNS is the noradrenaline/serotonin-reuptake inhibitor duloxetine. We know from a growing body of work that in addition to descending and local modulation of the spinal cord circuitry there exists a core network of brainstem, midbrain and cortical regions that are involved in the processing of pain. This core network has been referred to as the pain matrix, which includes primary and secondary somatosensory, insular, anterior cingulate and prefrontal cortices, as well as the brainstem, amygdala and cerebellum [11]. It is believed that variable levels of activity in these brain regions result in the conscious experience of different types of pain. Therefore, it is predicted that selective modulation of the pain-related activity in these areas could provide potent pain relief. However, these brain regions are also involved in a broad range of other important functions, from emotional processing to executive control and cognitive functions. Therefore, it will be crucial to identify specific molecular targets in the CNS pain circuitry that are relatively more important in processing pain signaling than processing of other signals. A second major challenge is that the currently validated preclinical methods to assess pain states in animal models may be biased to explore peripheral mechanisms and evoked pain, and therefore may not be sensitive enough to reveal meaningful pharmacological-induced alterations in spontaneous pain that may emerge from altered processing in the CNS [12]. In practice, the inability to demonstrate proof-of-concept data for a new target in a validated preclinical model is an impediment to the future development of novel potential analgesics in humans.

\section{Targeting key nodes or distributed mechanisms}

Chronic pain is a deceptively complicated experience in higher organisms. Superficially, chronic pain seems to be a simple stimulus-driven state 
that should be easy to treat by blocking the transduction of the stimulus. Instead, the growing body of knowledge indicates that chronic pain is a complex experience arising from the intricate interaction of external stimuli, the neurochemical and structural properties of the peripheral nervous system and CNS, the organism's prior experiences and the current emotional state. The brief discussion above and reviews cited therein illustrate that we know about many molecular targets involved in processing some aspect of pain in animals. However, the challenge that we face is to develop ways to predict which, if any, molecular mechanisms play a dominant role in the expression of pathological chronic pain in the clinic. In other words, we need to establish which of the targets are key nodes, either in biochemical pathways or in the pain circuitry, that integrate diverse pain signals or serve as common final pathways involved in pain signal processing. Of course, these key mechanisms, by their very nature, are likely to be important not just for processing chronic pain but also for other functions critical to the normal function of the organism. Therefore, while there is great promise in targeting key nodes for analgesic development, these targets must be adequately explored for the potential impact on normal sensory function that would limit their potential therapeutic usefulness.

\section{"Future advances in ... drug-development technology, including the advent of personalized medicine approaches, may be necessary to take advantage of a rational polypharmacy approach."}

An alternative to targeting a single molecular mechanism with a well defined role in the pain circuitry is to identify molecular targets that are broadly expressed and relatively enriched throughout the pain-processing circuitry. This approach exploits the possibility that interference at multiple sites within the pain circuitry will synergize to impact pain processing while minimizing the impact on other CNS functions, where the target may only play a minor role. The opioids and cannabinoid systems are known examples of this type of target because the ligands and receptors for these systems are expressed in the periphery, spinal cord and CNS and most likely achieve their efficacy by acting at all of these sites. Unfortunately, there are very few known molecular targets that fit this description.
A third and final approach to developing an effective therapy is to simultaneously engage a number of targets that provide synergy with respect to efficacy but not with respect to side effects. This can be achieved either by identifying a single multifunctional compound or by combining a collection of target-specific compounds that together result in at least additive, and perhaps synergistic, effects with respect to analgesic efficacy but not side effects. In fact, this approach finds support in the clinic, where it is common to prescribe multiple analgesics to achieve optimal treatment for any given patient. Indeed, some effective chronic pain therapies impact a variety of molecular targets. For example, the tricyclic antidepressant amitriptyline blocks sodium channels in addition to inhibiting serotonin and norepinepherine uptake; all three mechanisms probably contribute to analgesic efficacy. Unfortunately, many practical hurdles inhibit the identification and rational design of multifunction molecules or the development of combination formulations of new chemical entities. Future advances in lead identification, rational drug design and drug development technology, including the advent of personalized medicine approaches, may be necessary to take advantage of a rational polypharmacy approach to treat chronic pain.

In summary, many molecular targets are already known to be involved in the processing of chronic pain and more are likely to be discovered. However, the challenge remains to identify which of those many targets are key contributors to chronic pain as experienced by patients. Therefore, it is likely that the most high-impact future analgesic therapies will exploit innovative ways to broadly, and yet at the same time selectively, maximize their impact on pain circuitry.

\section{Acknowledgements}

Thanks to David Hewitt and Sean Cook for comments on this manuscript.

Financial \& competing interests disclosure Darrell Henze is an employee of Merck \& Co. Inc. and owns stock in the company. The author has no other relevant affliations or financial involvement with any organization or entity with a financial interest in or financial conflict with the subject matter or materials discussed in the manuscript. This includes employment, consultancies, honoraria, stock ownership or options, expert testimony, grants or patents received or pending, or royalties.

No writing assistance was utilized in the production of this manuscript. 


\section{Challenges faced in choosing novel targets to treat chronic pain}

\section{Bibliography}

1 Portenoy RK, Ugarte C, Fuller I, Haas G. Population-based survey of pain in the United States: differences among White, African American, and Hispanic subjects. J. Pain 5(6), 317-328 (2004).

2 Guindon J, Walczak JS, Beaulieu P. Recent advances in the pharmacological management of pain. Drugs 67(15), 2121-2133 (2007).

3 Marchand F, Perretti M, McMahon SB. Role of the immune system in chronic pain. Nat. Rev. Neurosci. 6(7), 521-532 (2005).

4 Millan MJ. The induction of pain: an integrative review. Prog. Neurobiol. 57(1), 1-164 (1999).
5 Patapoutian A, Tate S, Woolf CJ. Transient receptor potential channels: targeting pain at the source. Nat. Rev. Drug Discov. 8(1), 55-68 (2009).

6 Bingham B, Ajit SK, Blake DR, Samad TA. The molecular basis of pain and its clinical implications in rheumatology. Nat. Clin. Pract. Rheumatol. 5(1), 28-37 (2009).

7 Szallasi A, Cortright DN, Blum CA, Eid SR. The vanilloid receptor TRPV1: 10 years from channel cloning to antagonist proof-of-concept. Nat. Rev. Drug Discov. 6(5), 357-372 (2007).

8 Tai C, Zhu S, Zhou N. TRPA1: the central molecule for chemical sensing in pain pathway? J. Neurosci. 28(5), 1019-1021 (2008).
9 Waldmann R, Champigny G, Bassilana F, Heurteaux C, Lazdunski M. A proton-gated cation channel involved in acid-sensing. Nature 386(6621), 173-177 (1997).

10 England S. Voltage-gated sodium channels: the search for subtype-selective analgesics. Expert Opin. Investig. Drugs 17(12), 1849-1864 (2008).

11 Bingel U, Tracey I. Imaging CNS modulation of pain in humans. Physiology (Bethesda) 23371-23380 (2008).

12 Mogil JS, Crager SE. What should we be measuring in behavioral studies of chronic pain in animals? Pain 112(1-2), 12-15 (2004). 